\title{
Analysis of Chinese Entry-luxury Market and Research of Marketing Strategy
}

\author{
MI XUE \\ Tianjin University of Commerce, No. 2, Jinba Road East Side, Beichen District, Tianjin, China \\ 300134 \\ dearnina1992@163.com
}

Keywords: entry-luxury,marketing strategy , marketing analysis, consuming behaviour

\begin{abstract}
With the deceleration of Chinese economic development and the implementation of restraining luxury consuming policy, Chinese luxury market is definitely facing an "ice age" and the selling of luxuries in Chinese market has a sharp decline in 2014. At the same time, compared with the sluggish market of famous luxury brands, some entry-luxury brands become more and more popular among some consuming groups and the selling of these entry-luxury brands is steady increase. Based on the current condition of luxury market, the paper is primarily focused on the marketing management strategy of entry-luxury brands in Chinese local market by analyzing current situation of Chinese luxury consuming and the behaviors of Chinese luxury consumers.
\end{abstract}

\section{Introduction}

In order to introduce the origin of entry-luxury, the transition from Haute Couture to Ready-to-wear is the beginning ${ }^{[1]}$. Haute Couture which inherits the extravagant tradition of nobleman is indeed luxurious good, but because of the change of market demand and social culture, Haute Couture was gradually taken place by Ready-to-wear, losing the function of market wizard. Nowadays, the importance of product life cycle has been recognized by more and more companies. From 2011 to 2012 , almost all the fashion brands have tried their best to produce new designs and some brands such as ZARA , H\&M and C\&A have been particularly popular in the market. But with the popularity of these fast fashion brands, some problems have been found for example copying designs, bad quality and low produce cost.When consumers' requirements could not be entirely satisfied by the fast fashion products, entry-luxury is surly playing a more crucial role in the market.

Actually, the governmental policy is another essential factor to boost the development of entry-luxury. The selling of traditional luxury has been hampered by the "constraining luxury " policy published by Chinese government. Specifically, in Chinese market, consumers have been more rational and cautious in purchasing luxuries goods and some young consumers who would like to pay more attention to the meaning of a brand and the cost performance of a product, have generated relatively high purchasing power in luxuries.

However, for those consumers, traditional luxuries clearly can not fit their demands for luxury purchase, for a simple reason that the traditional luxuries' high price is not acceptable. While some other fast fashion brands can not provide the special design and good quality which these consumers are asking for. Thus, to set up some entry-luxury brands and construct the new selling pattern for entry-luxury is apparently meaningful and potential for all of the luxuries brands who seek better development in Chinese market after the change of market conditions.

\section{The analyses of current Chinese entry-luxury market}

With the slowing Chinese economy and Chinese government's "Clamp down on corruption" regulation, the turnover of luxuries goods has showed a sharp decline. According to the data provided by Bain Capital, the compound annual growth rate of Chinese luxuries selling was beyond 30\% in 2011; the sale volume growth in Chinese luxury market is $7 \%$ in 2012. While, in 
2013, the marketing increase rate in China is down to $4 \%$ and the growing rate of luxury market is only $2 \%{ }^{[2]}$.

The data from Bain Capital obviously points out that Chinese luxury market is gradually sinking into stagnancy and it is reasonable to speculate that this sluggish marketing performance will continue in 2014.

In August 2013, Chinese government made a clear policy banning the corruption caused by abusing government money, especially in sending gifts. Because of this policy, the fashion of buying luxuries for gifting has been extremely constrained and for some luxury companies, the policy is a fatal strike of selling and making profit in Chinese market. With the decline of the amount of regular consumers, the whole pattern of consuming luxuries has changed a lot, from only purchasing extremely expensive luxuries for a certain elite groups to considering the real function and specific design of the luxuries. Some luxury brands, in order to adapt to the marketing tread and occupy a better position in the coming competition, have already begun to exploit a new segmentation, the entry-luxury, with no logo or some new second line brands.

Exploiting entry-luxury market is possibly the first choice for some high-end luxury brands, especially when the traditional market has some troubles and probably can not be recovered in a short time. For these companies, they can make the use of their traditional brands to stimulate the development of entry-luxury brands and pursue the marketing diversity by enriching the marketing structure, and this can help luxury companies to take up market share and to gain profits.

\section{The analyses of consuming feature in China}

After the popularity of fast fashion from 2011 to 2012, especially when some disadvantages of these fast fashion brands such as copying design, bad quality and low cost price have been well-known by consumers, they become more rational toward the brand premium of luxuries ${ }^{[3]}$. Many consumers have changed their attitudes toward luxurious goods from only pursuing the well-know brand and the high price to paying more attentions to the meaning of a brand and the internal value of products. At the same time, consumers would like to pay a reasonable price for what they are pursuing-fashion, style and comfort. This stimulates the development of entry-luxury and for some consumers who want to buy luxurious things with lower price, they definitely have more flexible choices.

In China, the consumption of entry-luxury is primarily derived from two social groups, affluent class and white-collar workers. For affluent class, they always have high-level demands on living standard and they want to show their taste of fashion by buying the entry-luxury. For some white-collar workers, they are younger than the regular high-end luxury brand's consumers. According to the research, entry-luxury consumers takes up around $16 \%$ of the population in China , about 200,000,000 and the majority of consumers are living in first-tier cities and second-tier cities, with $¥ 10,000$ to $¥ 50,000$ income per year ${ }^{[4]}$. Furthermore, some of these consumers could be the same group of traditional luxuries consumers at certain times.Seriously, nowadays it is hard to distinguish the consumers of entry-luxury from the consumers of traditional luxuries and put them into a certain consumer group. Sometimes they may purchase the same kind of products, particularly when a brand have some branching brands attracts different consumers and blurs the bounds between luxury and entry-luxury.

However , the marketing positioning of entry-luxury is more clear than ever before and the number of consumers is constantly increasing with relative high brand loyalty.entry-luxury will play a crucial role in the foreseeable future and the prosperity of it is foreseeable. Thus, it is worthful for some marketing researchers to study the developing pattern and marketing strategy of entry-luxury.

\section{The marketing strategy of entry-luxury}

According to the analyses of entry-luxury market, there are several marketing managements of entry-luxury in Chinese market: 
(1)Pay more attention to the construction of brands' connotation and creating the resonance between consumers and brand culture. Most of entry-luxury brands are young brands and do not have rich brands' cultural heritage like traditional luxury brands. The main purchasing motivation of entry-luxury is that the consumers do agree with the brand connotation such as high living standard and elegant style provided by these brands ${ }^{[5]}$. Thus entry-luxury brands should have special cultural characters and set up the brand connotation which arouse the emotional resonance of consumers, for instance, a kind of good living attitude.

(2)Clarify brand positioning and avoiding the confusion of different brands. When a high-end luxury brand tries to exploit entry-luxury market by creating some new branching brands, it is critical to clarify the traditional brand with these new branching brands. For a simple reason that some alike brands may cause confusion and the mixture of different level brands may affect the traditional luxury brand's brand value. For example, the famous luxury brand Dolce \& Gabbana, was forced to close a branching brand $D \& G$, in order to keep the main brand's luxury degree,because similar brand positioning makes customers confuse about the main brand and the second line.

(3)Generate the channel strategy to protect the scarcity of entry-luxury. Although entry-luxury is a kind of "entry" level product, it still presents brand's value and specific design. If an entry-luxury brand can not find a suitable sale channel and sell in a big scale, it could become a common brand rather than a luxurious one. For entry-luxury, having a "limited-sale channel" strategy, without too many choices for consumers in the market and keeping a scarce selling situation, is crucial. Actually, with the rapid development in second-tier cities, entry-luxury brands should focus on the markets in these cities and build sale channels by setting up exclusive stores and cooperating with local department stores.

(4)Emphasis on the young consumers and use new media methods to advertise. With the development of the internet and media revolution, some old media have been extremely impacted. According to the research of China Economic Network , around 73\% consumers chose to use internet to obtain information about a luxurious good before really buying it. Thus entry-luxury should be tightly fasten to new media and make the use of media to advertise brand. There are several methods to advertise brand, weibo, wechat or even micro movies ${ }^{[6]}$. These new media have clear advantages, such as, low cost, fast spreading, good exchange ability and huge amount of audiences. This exactly fits the characters of entry-luxury, delivers the connotation of the brand well and builds a tight relationship with consumers ,especially the young ones.

(5)Cooperating with top-grade department stores and create a win-win situation. Some top-grade department stores are suitable sale channels for entry-luxury and a good pattern is building some "entry-luxury brand experience zones" to provide consumers diverse experiences with many kinds of different entry-luxury brands. This offers a chance for entry-luxury to show brands connotation and , at the same time, attract more consumers for department stores, help to make stores more famous in the local market, finally reaching the win-win situation.

\section{Summary}

To sum it up , there are some conclusions :

Firstly, with the change of policy and consuming characteristics, consumers are more rational in purchasing luxurious products and luxury brands can make good use of the conception "entry-luxury", to balance the designed style of luxury and the price. Furthermore, in order to take up more market share, luxurious brands and some branching brands should develop together to reach consumers' different demands.

Secondly, for entry-luxury , compared with traditional luxury brands, entry-luxury depends more on choice of marketing strategy. These entry-luxury brands should focus on constructing brand's connotation and setting the right brand position. And then, these brands should select some efficient media to advertise themselves after choosing the suitable media to balance the products' scarcity and distribution. 
Moreover, entry-luxury can collaborate with top-grade department stores by providing special experience zones and emphasizing the young consumers' importance, using flexible channel to advance their brand recognition among consumers, including by some traditional media and new media,especially some new media plat such weibo, weichat and micro movies.

\section{References}

[1] Cheng Mingyue: The Marketing Strategy of Fashion Luxury Brand in the New Economic Era[D]. Hu Nan: Hunan Normal University (2012).

[2] Information on http://www.ce.cn/

[3] Ding Jun: Entry-luxury’s Deep Marketing Experience[J].China Marketing (Management) No.8 (2012), p.54

[4] Zhu Ye: Entry-luxury Seize Market Share in China Market[N].China Fashion Weekly Volume No. A09, (8/6/2012)

[5] Massimiliano CIMS: Lux in China:Focus on Fashion and Luxury Industry in China[D]. Shang Hai: Fudan University (2011).

[6] Liang Xuemei: Luxury and New Media: Opportunity, Crisis and Future[J]. Southeast Communication Vol.12 (2012), p.37

Mi Xue(1992.9- ), born in Lan Zhou City, Gan Su Province, China. Mi Xue is an undergraduate student in Business School of Tian Jin University of Commerce, where she analyzes Marketing Strategy. 